\title{
Subdiagnóstico de baja densidad mineral ósea en mujeres post- menopáusicas
}

Identification and Fracture Outcomes of Undiagnosed Low Bone Mineral Density in Postmenopausal Women: Results of the National Osteoporosis Risk Assesment. Siris ES, Miller PD, Barrett-Connor E. JAMA 2001; 286: 2815.2822

\section{Objetivo}

Describir la ocurrencia de baja densidad mineral ósea en mujeres postmenopáusicas, sus factores de riesgo y la incidencia de fracturas a corto plazo.

\section{Diseño}

Estudio observacional longitudinal, de 12 meses de seguimiento.

\section{Lugar}

Participaron 34 estados de Estados Unidos de Norteamérica (4 236 médicos de atención primaria fueron derivadores).

\section{Pacientes}

200160 mujeres postmenopáusicas, ambulatorias, sin densitometría ósea en los últimos 12 meses, sin diagnóstico previo y sin tratamiento estándar para osteoporosis, excepto Terapia Hormonal de Reemplazo (THR).

Edad media: 64.5 años (DS: 9.3) con un rango de 20-104 años, $89.7 \%$ eran de raza blanca, $11 \%$ reportó al menos una fractura después de los 45 años, $11.7 \%$ con antecedentes maternos de osteoporosis, $63.2 \%$ habian usado o usaban THR y $45.1 \%$ eran o habían sido tabaquistas.

\section{Evaluación de factores pronósticos}

Se evaluaron potenciales factores de riesgo (tomados de publicaciones previas) para osteoporosis, a través de un autocuestionario individual (visita basal y a los 12 meses). El mismo incluía datos demográficos y los siguientes factores de riesgo: edad, raza, altura, peso corporal, edad a la menopausia, uso de THR, historia materna de osteoporosis, historia materna y personal de fracturas después de los 45 años, tabaquismo, autoevaluación del estado de salud, utilización de suplementos de calcio, de hormona tiroidea, de diuréticos ó de corticoides, consumo de café y alcohol. La tasa global de fracturas fue calculada por paciente y no por número total de fracturas. Se evaluó por densitometría periférica (pDEXA) de antebrazo y dedo, absorciometría de haz simple de rayos $\mathrm{X}$ de talón (SXA) y ultrasonido de calcáneo (US), la prevalencia de densidad mineral normal, osteopenia u osteoporosis según criterios de la Organización Mundial de la Salud.

\section{Medición de resultados principales}

Se analizaron los datos basales de 200160 mujeres y de 163979 mujeres $(81.9 \%)$ al año, se pudo establecer la prevalencia de diagnóstico densitométrico, prevalencia de fracturas clínicas e incidencia de fracturas a los 12 meses (en ambos casos por autoreporte).

\section{Resultados Principales}

Globalmente (considerando todos los equipamientos) la prevaIencia de osteopenia fue de $39.6 \%$ y de Osteoporosis fue $7.2 \%$, siendo ésta última diferente según la técnica empleada: $4.9 \%$ por SXA (OR 1 = referente), $3.4 \%$ por US (OR 0.79, IC 95\% 0.70-0.90), $10.2 \%$ por pDEXA antebrazo (OR 2.86 , IC $95 \% 2.75-2.99$ ) y $13.5 \%$ por pDEXA de dedo (OR 4.86, IC 95\% 4.56-5.18).

Del modelo multivariable* se identificaron los siguientes factores de riesgo independientes: edad avanzada, años desde la menopausia, pobre estado de salud, antecedentes personales de fractura, antecedentes maternos de fractura/osteoporosis, tabaquismo y uso de corticoides.

Fueron identificados como factores de menor riesgo de osteoporosis: Indice de Masa Corporal > $23 \mathrm{~kg} / \mathrm{m} 2$, uso de THR, uso de diuréticos y consumo de alcohol (1 a 6 bebidas por semana). La prevalencia global de fracturas osteoporóticas (radio, cuello femoral, o vértebras) fue de $11 \%$ y en el modelo de riesgo proporcional de Cox, la osteopenia se asoció a 1.73 veces más a riesgo de fractura y osteoporosis a 2.74 veces más a riesgo de fractura incidental, dentro del primer año. Los factores predictores independientes de fractura (con RH significativos) fueron los mismos que los asociadosa mayor riesgo de osteoporosis.

\section{Conclusión}

En mujeres postmenopáusicas que presentan factores de riesgo, estaría indicado la evaluación de la densidad mineral ósea.

Fuente de Financiamiento: Merck \& Co. Inc International Society for Clinical Densitometry (ISCD).

\section{Comentario}

Este estudio confirma la prevalencia de osteopenia/osteoporosis en la población americana ya descripta en estudios previos, como así mismo la importancia de los denominados factores de riesgo para osteoporosis. Llama la atención la prevalencia de fracturas osteoporóticas, en pacientes sin diagnóstico, ni tratamiento posterior de osteoporosis, lo cual indica una falta de conciencia en la comunidad médica acerca de las consecuencias de ésta enfermedad, sobre todo considerando que se excluían del estudio pacientes con diagnóstico conocido de osteoporosis, lo cual implica una subestimación de la realidad. Esto coincide con otros reportes en que aproximadamente sólo $1 / 5$ de los pacientes con fracturas osteoporóticas reciben tratamiento específico

Las limitaciones de éste estudio son principalmente la recolección de datos por autoreporte (lo cual conlleva a la pérdida de registro de fracturas vertebrales no clínicas, que son la mayoría) y también que se utilizaron distintos métodos densitométricos periféricos y ultrasónicos, que como se describió anteriormente, presentan capacidades discriminativas diferentes, poblaciones de control diferentes, además de ser métodos no definitivamente validados para diagnóstico final de osteoporosis, donde la densitometría central (DEXA) sigue siendo el estándar de referencia.

Hay que destacar, que la baja densidad mineral ósea es el principal factor de riesgo para padecer fracturas, pero que no es el único condicionante. Por lo tanto, estamos ante un evento multifactorial como lo demuestra la contradictoria ausencia de fracturas en osteoporóticas severas y la presencia de fracturas en mujeres con osteopenia moderada.

Conclusión del comentario: Considerando el alto riesgo de nuevas fracturas, es aconsejable intentar confirmar el diagnóstico y eventual tratamiento de osteoporosis, en pacientes con fracturas sugestivas (por localización y por trauma mínimo). Los métodos periféricos de diagnóstico tienen por ahora clara indicación sólo en el contexto de rastreo poblacional.

Dr. Fabio Massari [ Director Médico Dpto. Investigación Clínica IDIM Bs.As. Miembro Comisión Directiva Sociedad Argentina de Osteoporosis (SAO). Profesor Adjunto Maestría en Osteología y Metabolismo Mineral USAL ] 